\title{
Impact Of Interference On Multi-hop Wireless Network Performance
}

\author{
Kamal Jain Jitendra Padhye Venkata N. Padmanabhan Lili Qiu \\ Microsoft Research \\ One Microsoft Way, Redmond, WA 98052.
}

\{kamalj, padhye, padmanab, liliq\}@ microsoft.com

\begin{abstract}
In this paper, we address the following question: given a specific placement of wireless nodes in physical space and a specific traffic workload, what is the maximum throughput that can be supported by the resulting network? Unlike previous work that has focused on computing asymptotic performance bounds under assumptions of homogeneity or randomness in the network topology and/or workload, we work with any given network and workload specified as inputs.

A key issue impacting performance is wireless interference between neighboring nodes. We model such interference using a conflict graph, and present methods for computing upper and lower bounds on the optimal throughput for the given network and workload. To compute these bounds, we assume that packet transmissions at the individual nodes can be finely controlled and carefully scheduled by an omniscient and omnipotent central entity, which is unrealistic. Nevertheless, using ns-2 simulations, we show that the routes derived from our analysis often yield noticeably better throughput than the default shortest path routes even in the presence of uncoordinated packet transmissions and MAC contention. This suggests that there is opportunity for achieving throughput gains by employing an interference-aware routing protocol.
\end{abstract}

\section{Introduction}

Multi-hop wireless networks have been a subject of much study over the past few decades [1]. Much of the original work was motivated by military applications such as battlefield communications. More recently, however, some interesting commercial applications have emerged, such as "community wireless networks" $[2,28]$, and sensor networks [8].

A fundamental issue in multi-hop wireless networks is that performance degrades sharply as the number of hops traversed increases. For example, in a network of nodes with identical and omnidirectional radio ranges, going from a single hop to 2 hops halves the throughput of a flow because wireless interference dictates that only one of the 2 hops can be active at a time.

The performance challenges of multi-hop networks have long been recognized and have led to a lot of research on the medium access control (MAC), routing, and transport layers of the networking stack. In recent years, there has also been a focus on the fundamental question of what the optimal throughput of a multi-hop wireless network is. The seminal paper by Gupta and Kumar [14] showed that in a network comprising of $n$ identical nodes, each of which is communicating with another node, the throughput per node is $\Theta\left(\frac{1}{\sqrt{n \log n}}\right)$ assuming random node placement and communication pattern and $\Theta\left(\frac{1}{\sqrt{n}}\right)$ assuming optimal node placement and communication pattern. Subsequent work [10, 11, 20] has considered alternative models and settings, such as the presence of relay nodes and mobile nodes, and locality in inter-node communication, and their results are less pessimistic.

This paper also deals with the problem of computing the optimal throughput of a wireless network. However, a key distinction of our approach is that we work with any given wireless network configuration and workload specified as inputs. In other words, the node locations, ranges etc. as well as the traffic matrix indicating which source nodes are communicating with which sink nodes 
are specified as the input. We make no assumptions about the homogeneity of nodes with regard to radio range or other characteristics, or regularity in communication pattern. This is in contrast to previous work that has focused on asymptotic bounds under assumptions such as node homogeneity and random communication patterns.

We use a conflict graph to model the effects of wireless interference. The conflict graph indicates which groups of links mutually interfere and hence cannot be active simultaneously. We formulate a multi-commodity flow problem [4], augmented with constraints derived from the conflict graph, to compute the optimal throughput that the wireless network can support between the sources and the sinks. We show that the problem of finding optimal throughput is NP-hard, and present methods for computing upper and lower bounds on the optimal throughput.

We show how our methodology can accommodate a diversity of wireless network characteristics such as the availability of multiple non-overlapping channels, multiple radios per node, and directional antennas. We also show how multiple MAC protocol models as well as single-path and multi-path routing constraints can be accommodated.

We view the generality of our methodology and the conflict graph framework as a key contribution of our work.

To compute bounds on the optimal throughput, we assume that packet transmissions at the individual nodes can be finely controlled and carefully scheduled by an omniscient and omnipotent central entity. While this is clearly an unrealistic assumption, it gives us a best case bound against which to compare practical algorithms for routing, medium access control, and packet scheduling. Moreover, ns-2 simulations show that the routes derived from our analysis often yield noticeably better throughput than the default shortest path routes, even in the presence of real-world effects such as uncoordinated packet transmissions and MAC contention. In some cases, the throughput gain is over a factor of 2. The reason for this improvement is that in optimizing throughput, we tend to find routes that are less prone to wireless interference. For instance, a longer route along the periphery of the network may be picked instead of a shorter but more interference prone route through the middle of the network.

We use our technique to evaluate how the per-node throughput in a multi-hop wireless network varies as the number of nodes grows. Previous work (e.g., [14]) suggests that the per-node throughput falls as the number of nodes grows. But this result is under the assumption that nodes always have data to send and are ready to transmit as fast as their wireless connection will allow. In a realistic setting, however, sources tend to be bursty, so nodes will on average transmit at a slower rate than the speed of their wireless link. In such a setting, we find that the addition of new nodes can actually improve the per-node throughput because the richer connectivity provides increased opportunities for routing around interference "hotspots" in the network. This more than offsets the increase in traffic load caused by the new nodes.

The rest of this paper is organized as follows. In Section 2, we discuss related work. In Section 3, we present details of our conflict graph model and methods for computing bounds on the optimal network throughput. In Section 4, we present results obtained from applying our model to different network and workload configurations. In Section 5, we discuss ways to incorporate node mobility into our model. In Section 6 we discuss some limitations of our work. Section 7 concludes the paper.

\section{Related work}

A number of papers have been published on the problem of estimating the throughput of a multi-hop wireless network. Here, we consider the work that is most closely related to ours.

In their seminal paper [14], Gupta and Kumar studied the throughput of wireless networks under two models of interference: a protocol model that assumes interference to be an all-or-nothing phenomenon and a physical model that considers the impact of interfering transmissions on the signal-to-noise ratio. They show that in a network comprising of $n$ identical nodes, each of which is communicating with another node, the throughput per node is $\Theta\left(\frac{1}{\sqrt{n \log n}}\right)$ assuming random node placement and $\Theta\left(\frac{1}{\sqrt{n}}\right)$ assuming optimal node placement and communication pattern. These results are shown under the protocol model, but the latter result also holds in the case of the physical model under reasonable assumptions. According to the intuitive explanation in [20], while the overall one-hop throughput of the network grows as $O(n)$, the average path length grows as $O(\sqrt{n})$, so the throughput per node is $O\left(\frac{1}{\sqrt{n}}\right)$.

Li et al. [20] have extended the work of Gupta and Kumar [14] by considering the impact of different traffic patterns on the scalability of per node throughput. They 
point out that a random traffic pattern represents the worst case from the viewpoint of per-node throughput. They also show that for traffic patterns with power law distance distributions, the per-node throughput stays roughly constant as the network size grows, provided the distance distribution decays more rapidly than the square of the distance. Li et al. also consider the interactions of packet forwarding with the 802.11 MAC and show that the use of 802.11 instead of a global scheduling scheme does not affect the asymptotic bound on per-node throughput derived in [14].

In [11], Grossglauser and Tse introduce mobility into the model presented in [14], and show that the average long-term throughput per source-destination pair can be kept constant even as the number of nodes per unit area increases, provided that we allow for delays on the order of the time-scale of mobility. This is achieved by exploiting mobility to keep data transfers local, and transmitting only when the transmitter and receiver are close to each other, at a distance of $O\left(\frac{1}{\sqrt{n}}\right)$, thereby reducing total resource usage and interference. While this is encouraging, in many practical situations such as community wireless networks, mobility may be too infrequent or even nonexistent to be exploitable.

Gastpar and Vetterli [10] extend the work of Gupta and Kumar [14] in a different direction. Instead of the simple point-to-point coding assumption made in [14], which treats each transmitter-receiver pair as being independent of other pairs, they consider a network coding model where nodes could cooperate in arbitrary ways, for instance, to boost the transmit power. Further, they assume that there is a single source and single destination picked at random, and that the rest of the nodes act as relays. They show that the throughput of the network under these conditions is $O(\log n)$, compared to $O(1)$ for the point-to-point coding model of [14]. While the use of network coding in this context is a promising line of research, we note that the point-to-point coding model corresponds to current radio technology such as 802.11.

The recent work of De Couto et al. [5], based on two experiments in a $802.11 \mathrm{~b}$-based multi-hop wireless testbed shows that minimizing the hop count of an endto-end path is not sufficient for achieving good performance. The reason they point out is that link quality can vary widely and long hops may be included in "short" paths, resulting in a high packet error rate. In our work, we also reach the same conclusion regarding the lim- itations of the hop count metric, but for a somewhat different reason - because wireless interference limits throughput, a circuitous but less interference-prone route, say along the periphery of a network, may perform better than the shortest hop count route.

In [23], Nandagopal et. al. use a construct similar to conflict graphs, called flow contention graph to capture interference in wireless networks. However, as the name implies, the construct is defined on flows rather than on links. Moreover, the aim of that paper is to study MAC fairness issues, rather than to derive optimal throughput bounds.

Yang and Vaidya [29] also use the notion of a "conflict graph" in the context of their work on priority scheduling in wireless ad hoc networks. However, like [23], their conflict graph is also defined on flows rather than links. The graph is used only to interpret experimental results showing that the 802.11 MAC causes flows with a high degree of conflict to suffer disproportionately compared to flows with a low degree of conflict. There is no attempt to analyze the conflict graph to derive throughput bounds.

In [19], Kodialam and Nandagopal consider the problem of computing optimal throughput for a given wireless network with a given traffic pattern. They assume a limited model of interference in which the only constraint is that node may not transmit and receive simultaneously. With this constraint, they model the problem as a graph coloring problem. They provide a polynomial time algorithm that computes routes and schedules such that the resulting throughput is guaranteed to be at least $67 \%$ of the optimal throughput. The model we consider in this paper is much more general and flexible. Our model can take into account interference from neighboring nodes, impact of directional antennas, availability of multiple non-interfering channels etc. This generality makes the problem harder, so our algorithm only provides upper and lower bounds on optimal throughput.

We also note that our approach can compute the optimal throughput if we assume the limited model of interference assumed in [19]. See Appendix B for more details.

In summary, there is a large body of work on the multihop wireless throughput problem, much of it focused on asymptotic bounds under assumptions such as node homogeneity and random communication patterns. In contrast, our work focuses on computing throughput bounds for a given wireless network and traffic workload, using a conflict graph to model the constraints imposed by wire- 
less interference. We do not consider how factors such as mobility [11] or coding [10]. And like [14], we do not compute the information theoretic capacity of the network.

\section{Computing Bounds on Optimal Throughput}

We now present our framework for incorporating the constraints imposed by interference in a multi-hop wireless network and then present methods for computing bounds on the optimal throughput that a given network can support for a given traffic workload. We begin with some background and terminology.

\subsection{Background and Terminology}

Consider a wireless network with $N$ nodes arbitrarily located on a plane. Let $n_{i}, 1 \leq i \leq N$ denote the nodes, and $d_{i j}$ denote the distance between nodes $n_{i}$ and $n_{j}$. Each node, $n_{i}$, is equipped with a radio with communication range $R_{i}$ and a potentially larger interference range $R_{i}^{\prime}$. For ease of explanation, we start by considering the case of a single wireless channel. (We will generalize the model in Section 3.5.) We consider two models, the Protocol Model and the Physical Model, to define the conditions for a successful wireless transmission. These models are similar to those introduced in [14].

Protocol Model: In the protocol model, if there is a single wireless channel, a transmission is successful if both of the following conditions are satisfied:

1. $d_{i j} \leq R_{i}$

2. Any node $n_{k}$, such that $d_{k j} \leq R_{k}^{\prime}$, is not transmitting

Note that the second requirement implies that a node may not send and receive at the same time nor transmit to more than one other node at the same time. Note also that this model differs from the popular 802.11 MAC in an important way - it requires only the receiver to be free of interference, instead of requiring that both the sender and the receiver be free of interference. We discuss how to adapt the model for an 802.11-style MAC in Section 3.5.

Physical Model: Suppose node $n_{i}$ wants to transmit to node $n_{j}$. We can calculate the signal strength, $S S_{i j}$, of $n_{i}$ 's transmission as received at $n_{j}$. The transmission is successful if $S N R_{i j} \geq S N R_{\text {thresh }}$, where $S N R_{i j}$ denotes the signal-to-noise ratio at the node $n_{j}$ for transmissions received from node $n_{i}$. The total noise, $N_{j}$, at $n_{j}$ consists of the ambient noise, $N_{a}$, plus the interference due to other ongoing transmissions in the network. Note again that there is no requirement that the noise level at the sender also be low.

Our goal is to model wireless interference using a general framework that would enable us to compute the optimal throughput the wireless network can support for a given traffic workload. We assume that the workload consists of greedy sources and destinations, i.e. the sources always have data to send and the destination nodes are always ready to accept data. The communication between the sources and destinations can be either direct or be routed via intermediate nodes. We assume that packet transmissions at the individual nodes can be finely controlled and scheduled by an omniscient and omnipotent central entity.

We say that a network throughput $D$ is feasible if there exists a schedule of transmissions such that no two interfering links are active simultaneously, and the total throughput for the given source-destination pairs is $D$. In our problem formulation here, we focus on maximizing the total throughput between source-destination pairs.

In the rest of this section, we consider the following three scenarios in detail: (i) multipath routing under the protocol interference model, (ii) multipath routing under the physical interference model, and (iii) single-path routing under both models. We end the section by discussing several other generalizations, and summarizing our framework.

\subsection{Multipath Routing Under the Protocol In- terference Model}

Given a wireless network with $N$ nodes, we first derive a connectivity graph $C$ as follows. The vertices of $C$ correspond to the wireless nodes $\left(N_{C}\right)$ and the edges correspond to the wireless links $\left(L_{C}\right)$ between the nodes. There is a directed link $l_{i j}$ from node $n_{i}$ to $n_{j}$ if $d_{i j} \leq R_{i}$ and $i \neq j$. We use the terms "node" and "link" in reference to the connectivity graph while reserving the terms "vertex" and "edge" for the conflict graph presented in Section 3.2.1.

Let us first consider communication between a single source, $n_{s}$, and a single destination, $n_{d}$. In the absence of 
wireless interference (e.g., on a wired network), finding the maximum achievable throughput between the source and the destination, given the flexibility of using multiple paths, can be formulated as a linear program corresponding to a max-flow problem, as shown in Figure 1. Here, $f_{i j}$ denotes the amount of flow on link $l_{i j}, C a p_{i j}$ denote the capacity of link $l_{i j}$, and $L_{C}$ is a set of all links in the connectivity graph.

The maximization states that we wish to maximize the sum of flow out of the source. The first constraint represents flow conservation, i.e., at every node, except the source and the destination, the amount of incoming flow is equal to the amount of outgoing flow. The second constraint states that the incoming flow to the source node is 0 . The third constraint states that the outgoing flow from the destination node is 0 . The fourth constraint indicates the amount of flow on a link cannot exceed the capacity of the link. The final constraint restricts the amount of flow on each link to be non-negative.

Note that the above formulation does not take wireless interference into account. We turn to this issue next.

\subsubsection{Conflict Graph}

To incorporate wireless interference into our problem formulation, we define a conflict graph, $F$, whose vertices correspond to the links in the connectivity graph, $C$. There is an edge between the vertices $l_{i j}$ and $l_{p q}$ in $F$ if the links $l_{i j}$ and $l_{p q}$ may not be active simultaneously. Based on the protocol interference model described in Section 3.1, we draw such an edge if any of the following is true: $d_{i q} \leq R_{i}^{\prime}$ or $d_{p j} \leq R_{p}^{\prime}$. This encompasses the case where a conflict arises because links $l_{i j}$ and $l_{p q}$ have a node in common (i.e., $i==p$ or $i==q$ or $j==p$ or $j==q$ ). Note, however, that we do not draw an edge from a vertex to itself in the conflict graph.

Before we discuss how to use the conflict graph to add interference constraints in the linear program in Figure 1, we need to state a hardness result and a few definitions.

\subsubsection{Hardness Result}

We present a hardness result for computing the optimal throughput under the protocol interference model. Given a graph $H$ with vertex set $V_{H}$, an independent set is a set of vertices such that there is no edge between any two of the vertices. The independence number of graph $H$ is the size of the largest independent set in $H$. Then, we have the following hardness result.

Theorem 1 Given a network and a set of source and destination nodes, it is NP-hard to find the optimal throughput under the protocol interference model. Moreover, it is NP-hard to approximate the optimal throughput.

Proof : It can be shown that the problem of finding the independence number of a graph, which is a known hard problem even to approximate, can be reduced to the optimal throughput problem. Moreover, this reduction is approximation preserving. Hence the above hardness result. We describe the reduction in Appendix A.

Since it is NP-hard to approximate the optimal throughput, we now look at heuristics for obtaining lower and upper bounds on the optimal throughput. For this, we need to define some more terms. An independent set $I$ of a graph $H$ can be characterized using an independence vector, which is a vector of size $\left|V_{H}\right|$. This vector is denoted by $\mathbf{x}_{I}$. The $j^{\text {th }}$ element of this vector is set to 1 if and only if the vertex $v_{j}$ is a member of the independent set $I$, otherwise it is zero. We can think of $\mathbf{x}_{I}$ as a point in a $\left|V_{H}\right|$-dimensional space. The polytope defined by convex combination of independence vectors is called the independent set polytope or the stable set polytope.

\subsubsection{Lower Bound}

The problem of deriving a lower bound is equivalent to the problem of finding a network throughput $D$ that has a feasible schedule to achieve it. We make the following observation. Links belonging to a given independent set in conflict graph $F$ can be scheduled simultaneously. Suppose there are a total of $K$ maximal independent sets in graph $F$. A maximal independent set is one that cannot be grown further. Let $I_{1}, I_{2}, \ldots I_{K}$ denote these maximal independent sets, and $\lambda_{i}, 0 \leq \lambda_{i} \leq 1$ denote the fraction of time allocated to the independent set $I_{i}$ (i.e., the time during which the links in $I_{i}$ can be active). If we add the schedule restrictions imposed by the independent sets to the original linear program (Figure 1), the resulting throughput always has a feasible schedule, and therefore constitutes a lower bound on the maximum achievable throughput.

We formalize our above observation as follows. Given a conflict graph $F$, we define a usage vector, $U$, of size $\left|V_{F}\right|$, where $U_{i}$ denotes the fraction of time that the link 
$\max \sum_{l_{s i} \in L_{C}} f_{s i}$

\section{Subject To:}

$$
\begin{aligned}
\sum_{l_{i j} \in L_{C}} f_{i j} & =\sum_{l_{j i} \in L_{C}} f_{j i} & n_{i} \in N_{C} \backslash\left\{n_{s}, n_{d}\right\} & <1> \\
\sum_{l_{i s} \in L_{C}} f_{i s} & =0 & & <2> \\
\sum_{l_{d i} \in L_{C}} f_{d i} & =0 & & \\
f_{i j} & \leq \operatorname{Cap}_{i j} & \forall i, j \mid l_{i j} \in L_{C} & <4> \\
f_{i j} & \geq 0 & \forall i, j \mid l_{i j} \in L_{C} & <5>
\end{aligned}
$$

Figure 1: LP formulation to optimize the throughput for a single source-destination pair.

$i$ can be active. A usage vector is schedulable if the corresponding links can be scheduled, conflict free, for the fraction of the time indicated in the usage vector. If we think of the usage vector as a point in a $\left|V_{F}\right|$-dimensional space, we have the following theorem.

Theorem 2 A usage vector is schedulable if and only if it lies within the independent set polytope of the conflict graph.

Proof : Let us first show that a schedulable usage vector lies in the independent set polytope of the conflict graph. In other words, we want to show that the usage vector is a convex combination of independence vectors.

Consider a schedulable usage vector, $U$. Consider one unit of time, and assume that we have scheduled the links over fractions of this unit time, such that the usage vector has been satisfied. Since the vector is schedulable, such a schedule must exist. This schedule will tell us which links are active at any given instance of time. Also, since the usage vector is schedulable, at any instance in this schedule, the links that are active are not in conflict with each other. That is, the vertices corresponding to these links must form an independent set in the conflict graph. Find each such independent set $I$ and denote its independence vector by $\mathbf{x}_{I}$. Define $\lambda_{I}$ as the fraction of the unit time independent set $I$ is active. Since the total time is one unit, the sum of $\lambda_{I}$ 's over all the independent sets equals to one. Thus:

$$
U=\sum_{I \text { is an independent set }} \lambda_{I} \mathbf{x}_{I} .
$$

Now we show that a usage vector that is a convex combination of independence vectors is always schedulable. Consider a usage vector $U$ that is obtained by a convex combination of independence vectors:

$$
U=\sum_{I \text { is an independent set }} \lambda_{I} \mathbf{x}_{I}
$$

It follows that $U$ is schedulable since each independent set $I$ can be scheduled for $\lambda_{I}$ fraction of the time.

Theorem 2 implies that the optimal network throughput problem is a linear program, no matter how many sender-receiver pairs we have. In fact, the problem is that of maximizing a linear objective function over a feasible polytope. This feasible polytope can be described as the intersection of two polytopes - the flow polytope and the independent set polytope of the conflict graph. The flow polytope is the collection of feasible points described by the flow constraints (Figure 1), ignoring wireless conflicts. The flow polytope is a simple structure on which a linear objective function can easily be optimized. Independent set polytope, on the other hand, is a difficult structure and no simple characterization of it is known because there may be exponentially many independent sets. 
Theorem 2 implies that any convex combination of independence vectors is schedulable. In general, however, an arbitrary point inside the independent set polytope will be a convex combination of an exponentially many independence vectors. To get around this computational problem, we only want to pick "easy" points in the independent set polytope. An obvious notion of "easy" is that the point picked should be a convex combination of a small number of (i.e., polynomially many) independence vectors. We will be using this notion explicitly in the algorithm as follows. We derive a lower bound on the optimal throughput by finding $K^{\prime}$ independence vectors in the conflict graph $F$, and adding the following constraints to the LP formulation shown in Figure 1.

- $\sum_{i=1}^{K^{\prime}} \lambda_{i} \leq 1$ (because only one maximal independent set can be active at a time)

- $f_{i j} \leq \sum_{l_{i j} \in I_{i}} \lambda_{i} C a p_{i j}$ (because the fraction of time for which a link may be active is constrained by the sum of the activity periods of the independent sets it is a member of).

Note the solution produced by solving this linear program is always feasible (i.e., schedulable). This is due to the fact that all links belonging to independent set $I_{i}$ can be simultaneously active for $\lambda_{i}$ fraction of time, and we have required that the $\sum_{i=1}^{K^{\prime}} \lambda_{i} \leq 1$. Moreover, Theorem 2 assures us that when we include all independent sets, the solution will be exact, i.e., this will be the maximum value of $D$ that is feasible. To help tighten the lower bound more quickly, we should consider using maximal independence sets. While finding all maximal independent sets is also NP-hard [9], the lower bound obtained by considering a subset of the maximal independent sets has the nice property that as we add more constraints, the bound becomes tighter, eventually converging to the optimal (i.e., the maximum feasible) throughput when we add all the constraints.

\subsubsection{Upper Bound}

In this section, we derive an upper bound on the network throughput. Consider the conflict graph. A clique in the conflict graph is a set of vertices that mutually conflict with each other. Theorem 2 implies that the total usage of the links in a clique is at most 1 . This gives us a constraint on the usage vector. We can find many cliques and write corresponding constraints to define a polytope. We

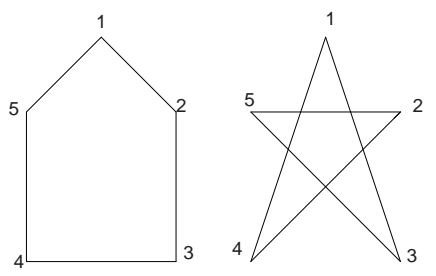

Figure 2: A pentagon and its complement graph. The former is an odd hole, and the latter is an odd anti-hole.

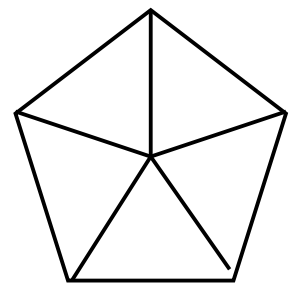

Figure 3: An example that shows it is not sufficient even if we add all clique, hole, anti-hole constraints.

can then maximize the throughput over the intersection of this polytope with flow polytope. This will give us an upper bound on the throughput.

Unfortunately, it is computationally expensive to find all the cliques, and even if we could find them all, there is still no guarantee that our upper bound will be tight. This can be illustrated by the following example. Suppose the conflict graph is the pentagon depicted in Figure 2. As we can see, the only cliques in the graph are formed by the adjacent pairs of nodes. Adding the clique constraints alone to the LP would suggest that a sum of link utilization equal to 2.5 is achievable. But actually at most 2 links can be active at a time. This suggests that we need to add constraints corresponding to odd holes and odd anti-holes. An odd hole is a cycle formed by an odd number of edges, without a chord in between. For example, the pentagon in Figure 2 is an odd hole. The sum of the link utilizations in an odd hole containing $k$ vertices can be no more than $\left\lfloor\frac{k}{2}\right\rfloor$. An odd anti-hole is the complementary graph of an odd hole. Figure 2 shows an example of an anti-hole with 5 nodes. The sum of link utilizations in an odd anti-hole can be no more than 2 .

Unfortunately, even if we consider the constraints imposed by the odd holes and odd anti-holes (in addition to those imposed by the cliques), we are not guaranteed to have a feasible solution. For example, consider the conflict graph, as shown in Figure 3. We can assign a uti- 
lization of 0.4 to all the vertices on the pentagon and 0.2 to the center of the pentagon, while satisfying all clique, hole, and anti-hole constraints. But there is no feasible schedule to achieve this, because this solution does not lie in the stable-set polytope. In fact, the upper bound based only on clique constraints is tight only for a special class of conflict graphs called perfect graphs. Perfect graphs are the graphs without any odd holes or odd anti-holes. Thus, in our present formulation, the upper bounds may not always be tight. We discuss this further in Appendix C.

\subsection{Multipath Routing Under the Physical In- terference Model}

As before, we begin by creating a connectivity graph $C$, whose vertices correspond to the nodes in the network. Based on the physical interference model, there exists a link, $l_{i j}$, from $n_{i}$ to $n_{j}$ if and only if $S S_{i j} / N_{a} \geq$ $S N R_{\text {thresh }}$ (i.e., the SNR exceeds the threshold at least in the presence of just the ambient noise).

Using the connectivity graph, we can write an LP formulation to optimize network throughput for a wired network. As discussed before, the solution to the linear program, as shown in Figure 1, provides an upper bound on network throughput. However, this bound is not very useful since it does not take interference effects into account.

To take interference effects into account, we construct a conflict graph $F$. Unlike in the protocol model, conflicts in the physical model are not binary. Rather, the interference gradually increases as more neighboring nodes transmit, and becomes intolerable when the noise level reaches a threshold. This gradual increase in interference suggests that we should have a weighted conflict graph, where the weight of a directed edge from vertices $l_{p q}$ to vertices $l_{i j}$ (denoted by $w_{i j}^{p q}$ ) indicates what fraction of the maximum permissible noise at node $n_{j}$ (for link $l_{i j}$ to still be operational) is contributed by activity on link $l_{p q}$ (i.e., node $n_{p}$ 's transmission to node $n_{q}$ ). Specifically, we have

$$
w_{i j}^{p q}=\frac{S S_{p j}}{\frac{S S_{i j}}{S N R_{\text {thresh }}}-N_{a}}
$$

where $S S_{p j}$ and $S S_{i j}$ denote the signal strength at node $n_{j}$ of transmissions from nodes $p$ and $i$, respectively, and $\frac{S S_{i j}}{S N R_{\text {thresh }}}-N_{a}$ is the maximum permissible interference noise at node $n_{j}$ that would still allow successful reception of node $n_{i}$ 's transmissions. The edges of the conflict graph are directed, and in general $w_{i j}^{p q}$ may not be equal to $w_{p q}^{i j}$.

\subsubsection{Lower Bound}

In the protocol model, we derive a lower bound on the network throughput by finding independent sets in the conflict graph $F$, and adding the constraints associated with the independent sets to the LP for the wired network. Analogous to independent sets, we introduce the notion of schedulable sets in the physical model. A schedulable set $H_{x}$ is defined as a set of vertices such that for every vertex $l_{i j} \in H_{x}, \sum_{l_{p q} \in H_{x}} w_{i j}^{p q} \leq 1$. It follows that all links in a schedulable set can be active simultaneously. Suppose we schedule the links belonging to $H_{x}$ for time $\lambda_{x}, 0 \leq \lambda_{x} \leq 1$. We now take the original LP for the wired network (in Figure 1), and include the following constraints:

- $\sum_{x=1}^{K^{\prime}} \lambda_{x} \leq 1$, where $K^{\prime}$ is the number of schedulable sets found

- $f_{i j} \leq \sum_{l_{i j} \in H_{x}} \lambda_{x} C a p_{i j}$

To tighten the bound, we should consider using maximal schedulable sets in graph $F$ (i.e., a schedulable set such that adding additional vertices to the set will violate the schedulable property). We have the following theorem, which is similar to the Theorem 2 in the protocol model.

Theorem 3 A usage vector is schedulable if and only if it lies in the schedulable set polytope of the conflict graph.

Proof : The proof is similar to that of Theorem 2.

\subsubsection{Upper Bound}

To derive an upper bound, we consider maximal sets of vertices in $F$ such that for any pair of vertices $l_{p q}$ and $l_{i j}$, $w_{i j}^{p q} \geq 1$. These correspond to the cliques in the protocol interference model. Therefore for each such set, we add a constraint that the sum of their utilization has to be no more than 1.

These constraints may result in a loose bound since there may not be very many cliques. To tighten the upper bound, we further augment the linear program with the following additional constraints. After we find a maximal schedulable set, say vertices $v_{1}, v_{2}, \ldots, v_{t}$, adding 
any additional vertex, denoted as $v_{a}$, to the set will make the set unschedulable. Therefore we have the following constraint: $U_{1}+U_{2}+\ldots U_{t}+U_{a} \leq t$, where as before $U_{i}$ denotes the fraction of time for which physical link $l_{i}$ (corresponding to vertex $v_{i}$ in the conflict graph) is active. By adding as many such constraints as possible, we can tighten the upper bound. Still, the bound is not guaranteed to converge to the optimal even if we include all such sets.

\subsection{Single-path Routing}

So far we have considered multipath routing. As many existing routing algorithms $[17,27,26,25]$ are confined to single-path routing, it is useful to derive a throughput bound for single-path routing so that we can compare how much the current protocols deviate from the theoretical achievable throughput under the same routing restriction. The way we enforce the single-path restriction for the flow from a source to a destination is by adding the following additional constraints to the LP problem for the wired network (shown in Figure 1):

- For each link $l_{i j}, f_{i j} \leq C a p_{i j} \cdot z_{i j}$, where $z_{i, j} \in$ $\{0,1\}$

- At each node $n_{i}, \sum z_{i j} \leq 1$

Here $z_{i j}$ is a $0-1$ variable that indicates whether or not link $l_{i j}$ is used for transmissions, and $f_{i j}$ is the amount of flow on the link. The basic intuition for these constraints is that in a single-path routing, at any node in the network, there is at most one out-going edge that has a non-zero flow. Since $z_{i j}$ can have only one of two values, either 0 or 1 , the two conditions ensure that at node $n_{i}$ at most one $z_{i j}$ will have a value of 1 .

In theory, solving integer linear program is a NPhard [9], but in practice, software such as lp_solve [3] and CPLEX [6] can solve mixed-integer programs.

\subsection{Other Generalization}

The basic conflict graph model is quite flexible, and can be generalized in many ways.

- Multiple source-destination pairs: We can extend our formulations in the previous sections from a single source-destination pair to multiple sourcedestination pairs using a multi-commodity flow formulation [4] augmented with constraints derived from the conflict graph. We assign a connection identifier to each source-destination pair. Instead of the flow variables $f_{i j}$, we introduce the variable $f_{i j k}$ to denote the amount of flow for connection $k$ on link $l_{i j}$. Referring to Figure 1, the flow conservation constraints at each node apply on a per-connection basis (constraint $<1>$ ); the total incoming flow into a source node is zero only for the connection(s) originating at that node (constraint $<2>$ ); likewise, the total outgoing flow from a sink node is zero only for the connection(s) terminating at that node (constraint $<3>$ ); and the capacity constraints apply to the sum of the flows over all connections traversing a link (constraint $<4>$ ).

- Multiple wireless channels: It may be the case that instead of just one channel, each node can tune to one of $M$ channels, $M \geq 1$. This can be easily modeled by introducing $M$ links between nodes $i$ and $j$, instead of just 1 . In general, links corresponding to different channels do not conflict with each other, reflecting the fact that the channels do not mutually interfere. However, the links emanating from the same node do conflict, reflecting the constraint that the single radio at each node can transmit only on one channel at a time.

- Multiple radios per node: Each wireless node may be equipped with more than one radio. If each node has $M$ radios, this can be modeled by introducing $M$ links between each pairs of nodes. If we assume that each of these radios is tuned to a separate channel, and that a node can communicate on multiple radios simultaneously, then the conflict graph will show no conflict among the $M$ links between a pair of nodes.

- Directional antennas: We can combine the use of directional antennas with the basic protocol model of communication. Instead of specifying a range for each node, we simply specify a list of nodes (or points in space) where transmissions or interference from this node can be perceived. The connectivity graph and the conflict graph are modified to take this into account.

- Multirate radios: Many wireless technologies support multirate radios, which can switch between a set of discrete data rates depending on the quality of 
the RF channel. For instance, 802.11b supports 4 rates: $1,2,5.5$, and $11 \mathrm{Mbps}$. We can model this in our framework by creating multiple "virtual" links corresponding to a physical link in the connectivity graph, one for each rate. The conflict graph is augmented to reflect the fact that only one of the virtual links corresponding to a physical link can be active at a time. The weights assigned to the edges of the conflict graph (under the physical interference model) would reflect the specific noise tolerance of the virtual link corresponding to each rate.

- Multiple transmit power levels: We have thus far assumed that transmitters used a fixed power level. However, we can extend our framework to incorporate a discrete set of transmitter power levels. We create multiple "virtual" links corresponding to each physical link in the connectivity graph, one for each power level. Depending on the environment and the proximity between the transmitter and the receiver, the different power levels may also correspond to different modulation schemes and/or different data rates. Using the physical model, we create edges in the conflict graph whose weights are a function of the power level of the links in the connectivity graph. This would, for instance, allow modeling of the case where a transmitter communicating with a nearby receiver switches to a lower power, while perhaps still maintaining a high data rate, given the proximity of the nodes, to minimize interference on other nodes.

- Other models of interference: In the simple example, we considered an optimistic model of interference that did not require the sender to be free of interference. But a more realistic model, which more closely reflects the situation in 802.11 , would require both the sender and the receiver to be free of interference. This reflect the fact that 802.11 may perform virtual carrier sensing using an RTS-CTS exchange, and that for successful communication, the sender must be able to hear the link layer acknowledgment transmitted by the receiver. Therefore, we draw an edge in the conflict graph between vertices $l_{i j}$ and $l_{p q}$ if $d_{a b} \leq R_{a}^{\prime}$ for $a b=$ $i q, q i, i p, p i, j p, p j, j q$, or $q j$.

- Non-greedy sources or destinations: We can easily accommodate the case where the rate at which nodes generate data or are willing to accept data is bounded. We do so by creating a virtual source or sink node and connecting it to the real source or sink via a virtual link of speed equal to the source or sink rate. The virtual link is special in that it is assumed not to interfere with any other link in the network. The virtual link is just a convenient construct to help us model the bound on the source or sink rate.

- Other objective functions: Our framework is not limited to maximizing the total network throughput. We can accommodate any objective that can be expressed as a linear function. For example, we can assign a linear revenue function to each sourcedestination pair, and then maximize the revenue instead of maximizing the total network throughput. We can also maximize the minimum throughput across all source-destination pairs, to provide a degree of fairness.

Many of these generalizations can be combined with each other to model complex networking scenarios. We will see examples of such combinations in Section 4.

\subsection{Summary}

In this section, we presented the concept of a conflict graph, and discussed how it could be used to derive upper and lower bounds on the optimal throughput that a wireless network can support, for a given set of sources and destinations. We show that the conflict graph model can be generalized to handle a wide range of scenarios. We have shown that the lower bound derived from our framework is always schedulable, and will be optimal once all the independent set constraints are incorporated. If the upper and lower bounds are equal, then these correspond to the optimal solution. We have not dealt with the question of node mobility so far, but we will present some ideas in Section 5.

\section{Results}

This section presents several results based on our model. The section is organized as follows. In Section 4.1, we present illustrative results that demonstrate the flexibility of our model. In Section 4.2, we use our model to provide insights into the tradeoff between the richer connectivity provided by the increase in the size of a wireless mesh 
network and the increase in cumulative traffic load to the new mesh participants. In Section 4.3, we i trate how optimal routing can bring benefits even ir sence of optimal scheduling (i.e., in the presence of $\Lambda$ contention and other inefficiencies). In Section 4.4 discuss the issue of convergence of the upper and l, bounds to the optimal throughput. Finally, in Section we present a discussion of the computational costs 0 . model.

\subsection{Illustrative Results}

In this section, we present several illustrative resul demonstrate the capabilities of our model. We begi defining a metric for computational effort. In Section 3, we have described the procedure for finding upper and lower bounds on throughput. Let us consider the protocol model of interference, and focus on the lower bound. We have shown that as we include more distinct independent sets, the lower bound becomes progressively tighter. In other words, the more effort we spend looking for independent sets in our conflict graph, the better the bound will be. Since we can not always hope to find optimal solutions, any upper or lower bounds discovered by our model need to be presented along with the amount of effort required to find those bounds. Thus we require a metric to measure this effort. We use the following simple algorithm to find distinct independent sets:

1. Start with an empty independent set $I S$.

2. Consider a random ordering of vertices in the conflict graph.

3. Consider the vertices of the graph in that order. Always add the first vertex to $I S$.

4. Add a new vertex if and only if it does not have an edge to any of the vertices added to $I S$ so far. Once we consider all the vertices, $I S$ will be of size at least one.

5. We check to see if we have previously discovered this independent set, and if not, we add constraints based on this independent set to our linear program. Otherwise we discard the set.

We consider this entire sequence as one unit of effort. Note that one unit of effort does not always result in addition of a constraint or variable to the linear program.

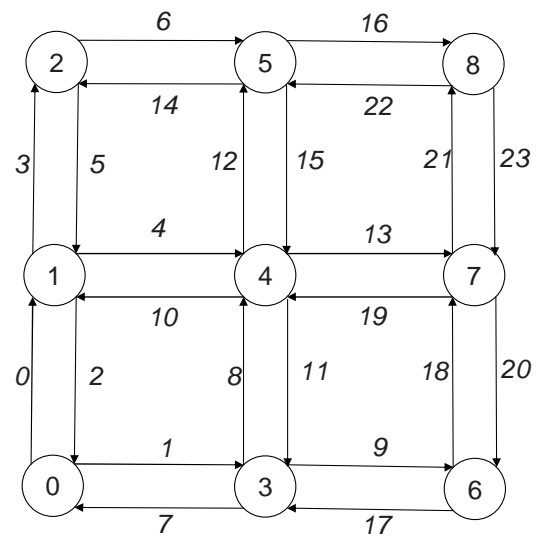

Figure 4: 3x3 Grid

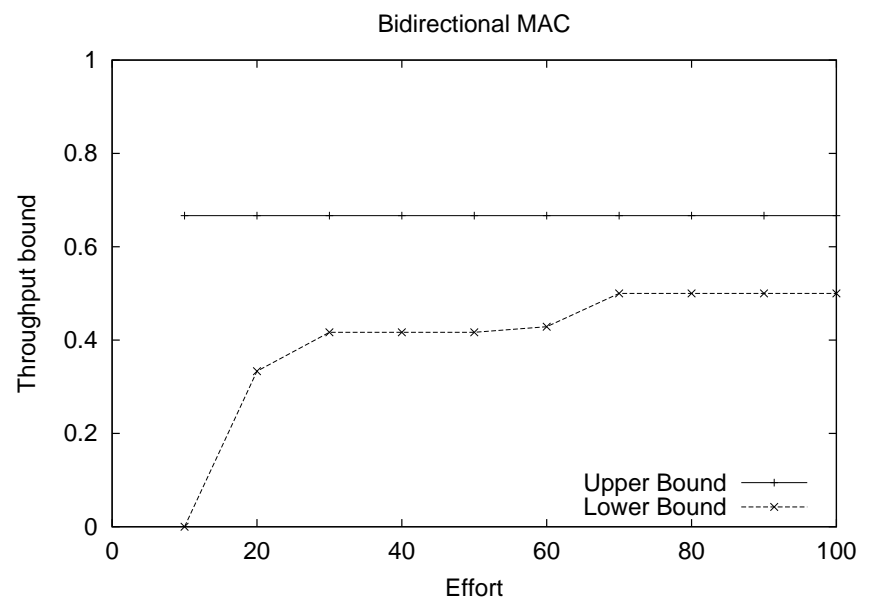

Figure 5: Throughput with a bidirectional MAC

Moreover, there is a complex relationship between the number of variables and constraints in a linear program, and the amount of time required to solve it. Thus, the metric is only a rough guide for the amount of actual time (or CPU cycles) spent while finding the bound. In Section 4.5, we will provide further discussion about the relationship between the effort metric and actual time spent in computation. The effort metric is defined in a similar manner by considering cliques in case of searching for the upper bound, and by considering schedulable sets in case of the physical model.

\subsubsection{A Simple Topology}

We consider the topology shown in Figure 4. The network consists of 9 nodes, placed in a $3 \times 3$ grid. We make no claims that this topology is representative of typical wireless networks. We have deliberately chosen a small, 


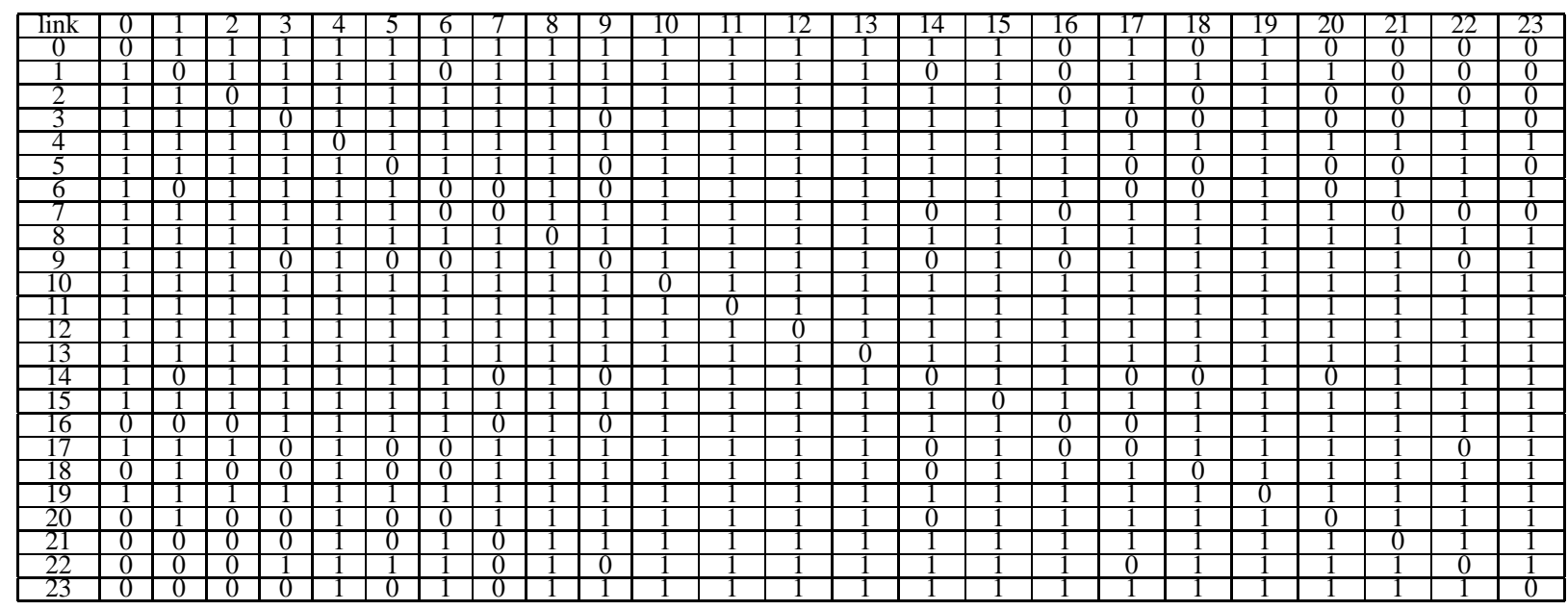

Table 1: Conflict Graph in matrix form

simple topology, to facilitate detailed discussion of the results.

We start with several simplifying assumptions. We will relax these assumptions as we proceed through the section. We assume that the range of each node is one unit, i.e., just enough to reach its lateral neighbors, but not the diagonal ones. We also assume that the interference range is equal to the communication range. We assume an 802.11-like protocol model of interference described in Section 3.5. This model requires both the sender and the receiver to be free of interference for successful communication. We term this a bidirectional MAC. The resulting conflict graph for this scenario is shown in the matrix form in Table 1. A 0 indicates that the links are not in conflict with each other, while 1 indicates otherwise. For example, when node 0 is transmitting to node 3 , node 1 can hear these transmissions, and hence can not transmit to node 2 . Thus, links $1(0 \rightarrow 3)$ and $3(1 \rightarrow 2)$ are in conflict.

We allow multipath routing. We assume that all wireless links have an identical capacity (i.e., speed) of 1 unit and that all nodes have infinite buffers. We designate node 0 to be the sender, and node 8 to be the receiver. The sender always has data to send, and the receiver is always willing to consume the data.

In this scenario, it is easy to see that the optimal throughput is 0.5. A convenient way to visualize the optimal transmission schedule is to imagine that time is divided into slots of equal size, and in each slot we can transmit one packet between neighboring nodes, subject to constraints imposed by the conflict graph. Then, the following transmission schedule will achieve optimal throughput: (i) $0 \rightarrow 1$ (ii) $1 \rightarrow 2$ (iii) $0 \rightarrow 3$ and $2 \rightarrow 5$ (iv) $3 \rightarrow 6$ and $5 \rightarrow 8$ (v) $0 \rightarrow 1$ and $6 \rightarrow 7$ (vi) $\ldots$ We can continue in this manner indefinitely. It is easy to see that in alternate timeslots, node 0 gets to transmit to either node 1 or 3 . Hence the optimal throughput is 0.5 .

In Figure 5, we show the upper and lower bound on throughput calculated by our model, as we devote increasing amount of effort. As shown, the upper bound quickly converges to the stable value of 0.667 , which is somewhat higher than the optimal value. This is a clear indication of the fact that clique constraints alone are not sufficient to guarantee optimality, even in such a small graph, as noted in Section 3.2.4. The lower bound, on the other hand, steadily converges to the optimal value of 0.5 . We have verified that our program has discovered all independent sets and cliques with 100 units of efforts.

\subsubsection{Community Networking Scenario}

Our model can also incorporate single path routing, multiple source-destination pairs, multiple channels as well as multiple radios. We demonstrate this flexibility with a community mesh networking scenario, in which multiple users share an Internet connection, using a multihop wireless network. We consider a map of a real suburban neighborhood shown in Figure 6. There are 252 houses in an area of 1 square kilometer. We select 35 of these houses at random, and assume that these houses are equipped with hardware that enables them to participate in a wireless mesh network. We assume that communication range of the wireless technology is 200 


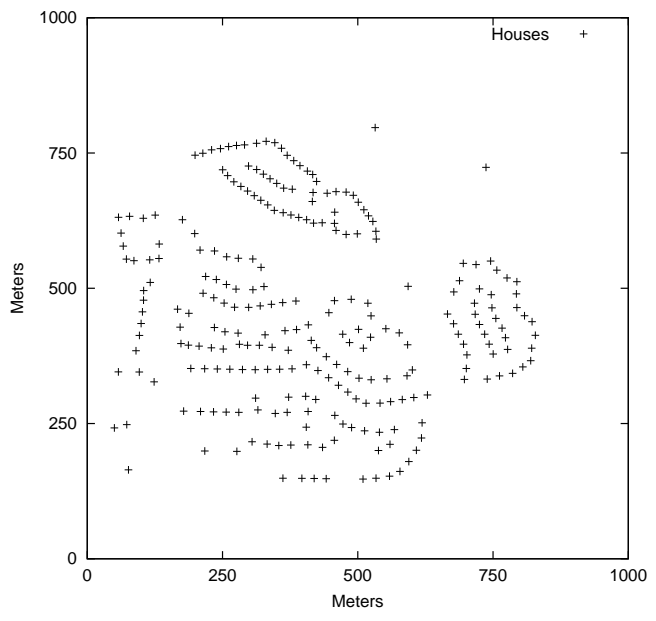

Figure 6: Neighborhood Map

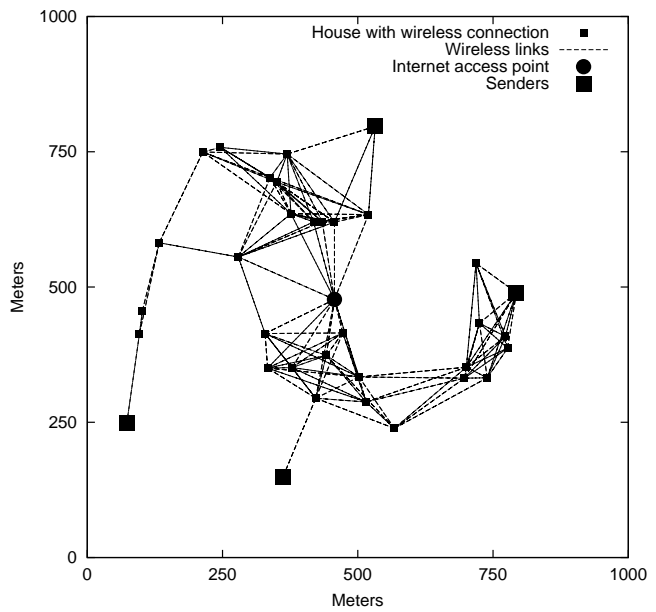

Figure 7: Mesh formation in the neighborhood

\begin{tabular}{|r|r|}
\hline Scenario & Optimal Throughput \\
\hline I & 0.5 \\
\hline II & 0.5 \\
\hline III & 1 \\
\hline IV & 1 \\
\hline
\end{tabular}

Table 2: Throughput for neighborhood mesh in various scenarios

meters, while the interference range is 400 meters. In Figure 7, we show the resulting network. We select a node that is roughly at the center of the area and designate it as the Internet access point. We assume that there are four senders, located as shown in the Figure. All the senders communicate with the Internet access point, and the metric of interest is the cumulative throughput of these senders. We assume that all wireless links are of unit capacity.

We begin with a baseline case, for which we assume a bidirectional MAC and single path routing. Our linear program is set to optimize the sum of the throughputs of the four flows, with no consideration of fairness. In this case, with about 5000 units of effort, upper and lower bounds converge, and our model indicates that the maximum possible cumulative throughput is 0.5 .

We may now ask what we can do to improve the cumulative throughput. We consider four possibilities: (I) Employ multi-path routing. (II) Double the range of each radio. We also double the interference range. (III) Leave the radio range unchanged, but use two non-overlapping channels instead of one. A node may communicate on only one of the two channels at any given time, but may switch between channels as often as necessary. (IV) Use two radios instead of one at each node. The radios are assumed to be tuned to two fixed, non-overlapping channels, so a node may communicate on the two channels simultaneously. The throughput bounds in each of the four scenarios are shown in Table 2. In each case, the upper and the lower bounds converge to the same value, which indicates that the solution is optimal.

The results indicate that neither multipath routing nor doubling the range of the radio increases cumulative throughput in the scenario we considered. On the other hand, by using two channels instead of one, the network may achieve the maximum possible throughput of 1 . The maximum possible throughput is 1 because the Internet access point has only one radio. On the other hand, even if we use two radios, the throughput remains at one. It is 
not hard to see why. The situation is equivalent to having two separate copies of the baseline network, and then adding up their throughputs. These scenarios illustrate that the model we have developed can be used as a tool for analysis and capacity planning of wireless multi-hop networks.

\subsection{Tradeoff Between Connectivity and Throughput}

In Section 3, we discussed how our model can accommodate nodes which do not send data in a greedy fashion, i.e. they have a lower send rate. In $[15,20]$, the authors have shown that the per node throughput in the network decreases as the number of nodes in the network goes up. These results, however, were derived under the assumption that each node sends data as fast as it can. In other words, the desired sending rate of the node is assumed to be 1 . However, if each node has a lower desired sending rate, the richer connectivity provided by additional nodes might help increase per node throughput, by allowing better routes to be discovered. We now explore this hypothesis using our model.

We consider a 7x7 grid, whose nodes are 200 meters apart horizontally, and vertically. We assume that the communication range is 250 meters, and the interference range is 500 meters. We set the link capacity to 1 . We assume a bidirectional MAC, similar to the one used to plot Figure 5. We use single-path routing.

We pick $\mathrm{N}$ nodes from the 49 available nodes, at random, and without replacement. Half of these nodes are designated as senders, and the other half are designated as receivers. The senders and the receivers form $N / 2$ flows in the network. Each sender is paired with only one receiver. We first calculate the fraction of flows for which the source and the destination lie in the same connected component of the topology. We call this fraction the connectivity ratio. The connectivity ratio for various values of $N$ is shown in Figure 8. The results show that after 24 nodes (i.e. 12 flows) are selected, the connectivity ratio becomes 1 .

We then assign a sending rate of $D$ to each sender. Then, using our model, we calculate the optimal throughput using single-path routing. We divide the cumulative throughput by the number of flows (i.e. $N / 2$ ) to obtain average per-flow throughput, and normalize it further by dividing it by $D$. The resulting normalized per-flow throughput for various values of $N$ and $D$ is plotted in

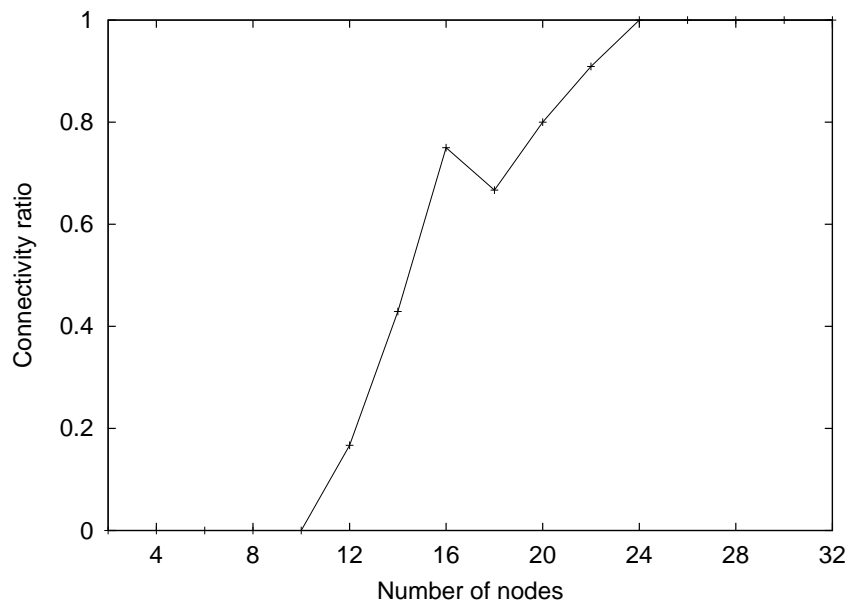

Figure 8: Connectivity Ratio for 7x7 grid

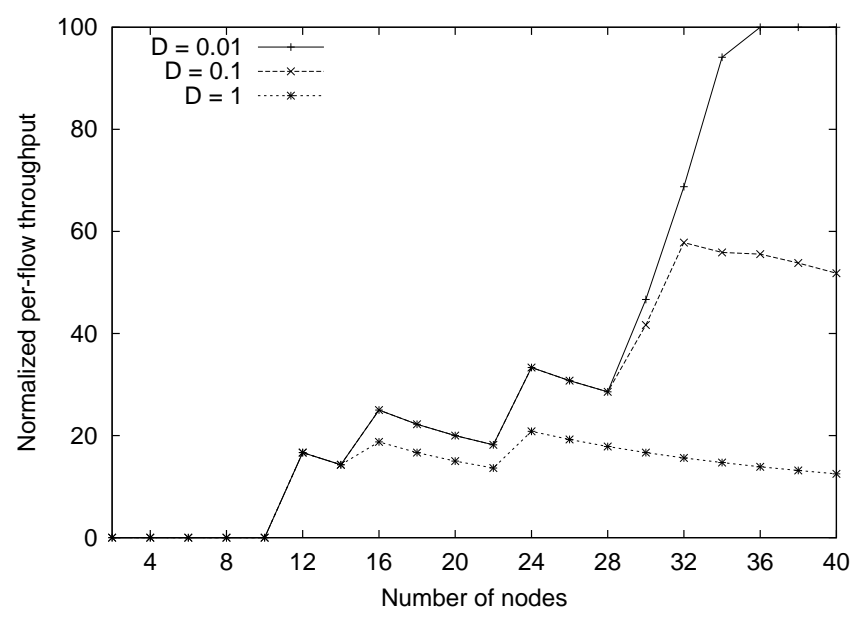

Figure 9: Normalized per-flow throughput

Figure 9.

Note that when the sending rate is 0.01 , the normalized per-flow throughput continues to rise even after the connectivity has reached 1 . This means that the richer connectivity provided by additional nodes allows for newer routes, and allows extra traffic to be sent through the network. However, if each node sends at rate 1, the node might have little capacity left to forward traffic from other nodes. Thus, the average per-flow throughput peaks early (i.e the network is saturated), and then declines slowly, as new nodes join the network, but fail to transmit most of their desired traffic. For sending rate of 0.1, the results are between these two cases. Note that the non-monotonic nature of the graphs is due to fluctuation in random runs. As part of our future work, we plan to 


\begin{tabular}{|l|l|l|}
\hline \multicolumn{1}{|c|}{ Scheduling } & Optimal & 802.11 \\
\hline Optimal & $\begin{array}{l}\text { Optimal } \\
\text { throughput } \\
\text { (solve LP) }\end{array}$ & $\begin{array}{l}\text { Alternative } \\
\text { routing scheme } \\
\text { (ns simulation) }\end{array}$ \\
\hline Shortest-path & $\begin{array}{l}\text { State of art } \\
\text { under optimal } \\
\text { scheduling } \\
\text { (solve LP) }\end{array}$ & $\begin{array}{l}\text { State of art } \\
\text { (ns simulation) }\end{array}$ \\
\hline
\end{tabular}

Figure 10: Four scenarios.

verify the generality of this result using a wide variety of topologies.

We stress that these results have been derived by assuming optimal routing, as well as optimal scheduling of packets. In the next section, we further discuss the impact of these two assumptions.

\subsection{Benefits of Optimal Routing in Absence of Optimal Scheduling}

As shown in the previous sections, the optimal throughput is achieved by selecting optimal routes and scheduling the links on the routes appropriately. A natural question to ask is how much performance improvement is due to the optimal route selection, and how much is due to the optimal scheduling. Motivated by this question, we empirically examine four scenarios shown in Figure 10. They correspond to (i) optimal routing with optimal scheduling, (ii) shortest-path routing with optimal scheduling, (iii) "optimal" routing under 802.11 MAC ${ }^{1}$, (iv) shortest-path routing under 802.11 MAC. We first briefly describe the approach we use to derive throughput for each case, and then present the results.

Given a network topology, we apply the algorithm described in Section 3 to compute the optimal throughput under single-path routing. This corresponds to scenario (i).

To derive the performance of optimal routing under 802.11 , we run $n s-2$ [24] simulations. To ensure that the packets follow the optimal routes, we specify the optimal routes obtained in Scenario (i) as the static routes

\footnotetext{
${ }^{1}$ This means routes derived in (i) used with 802.11 MAC. It may also be possible to derive optimal routes for contention-based scheduling, but that is not our intent here.
}

in ns-2. The throughput numbers from these simulations correspond to scenario (iii).

We then repeat our simulation using AODV [27], a standard shortest path routing protocol. The resulting throughput corresponds to the performance of the scenario (iv). To minimize the impact of AODV routing overhead, all nodes are static and simulations are run for 50 seconds, long enough to make the initial route setup overhead negligible.

Based on the AODV simulation results, we obtain a set of links that are used in the shortest paths between sources and destinations. We then modify the LP formulation in Section 3 to compute bounds on the optimal throughput by excluding all but those links that lie on one or more of the shortest paths. We do so by setting the capacity of the excluded links to zero. We solve the resulting LP, and obtain the throughput for scenario (ii).

Our aim is to compare throughput in scenario (i) to throughput in scenario (ii). Similarly, we compare scenarios (iii) and (iv) against each other. Note that we do not compare the throughput obtained by solving the LP model with the throughput obtained from ns-2 simulations.

We consider these four scenarios in a $7 \times 7$ grid (49 nodes). The horizontal and vertical separation between adjacent nodes is 200 meters. We assume the communication range to be 250 meters, and the interference range to be 500 meters. All other parameters are at their default settings in $n s-2$. For each simulation run, we randomly pick a few pairs of nodes as sources and destinations; the source sends packets to the corresponding destination at a constant bit rate equal to the wireless link capacity.

Table 3 shows the throughput ratios between optimal routing and shortest path routing, under optimal scheduling. These numbers are derived from our LP formulation. In all cases, optimal routing yields comparable or better throughput than the shortest path routing when optimal scheduling is used. The benefit of optimal routing varies with the number of flows, as well as with the locations of communicating nodes. For instance, when the two flows are far apart and do not interfere with each other, the optimal path achieves the same throughput as the shortest path (e.g., numFlow $=2$ and run=1,5); when the two flows interfere with each other, the optimal path takes a detour, which results in reduced interference and hence higher throughput (e.g., the case of numFlow $=2$ and $r u n=2,3$, 4).

Table 4 shows the throughput ratios between "optimal" 


\begin{tabular}{|c|c|c|c|c|c|}
\hline numFlow & run 1 & run 2 & run 3 & run4 & run 5 \\
\hline 2 & 1.00 & 1.25 & 1.60 & 1.38 & 1.00 \\
\hline 4 & 1.41 & 1.00 & 1.44 & 1.43 & 1.14 \\
\hline 8 & 2.10 & 1.00 & 1.05 & 1.11 & 1.11 \\
\hline
\end{tabular}

Table 3: Throughput ratios between optimal routing and shortest path routing, both under optimal scheduling in a $7 \times 7$ grid.

\begin{tabular}{|c|c|c|c|c|c|}
\hline numFlow & run 1 & run 2 & run 3 & run4 & run 5 \\
\hline 2 & 1.08 & 2.43 & 1.53 & 1.80 & 1.19 \\
\hline 4 & 1.07 & 1.54 & 0.79 & 1.02 & 1.55 \\
\hline 8 & 3.55 & 1.22 & 0.50 & 1.14 & 0.40 \\
\hline
\end{tabular}

Table 4: Throughput ratios between "optimal" path routing and shortest path routing, both under 802.11 MAC in a $7 \times 7$ grid.

routing and shortest path routing, under under the 802.11 MAC. These numbers are based on ns-2 simulations. Optimal path outperforms the shortest path even under the 802.11 MAC when number of flows in the network is small. On the other hand, the optimal path routing does not always outperform the shortest path routing under 802.11 MAC when the number of flows is higher. This occurs because as network load increases, it is harder to find paths that do not interfere with other flows in the absence of optimal scheduling.

The above results are encouraging, and suggest that there is a potential to improve throughput by making route selection interference-aware. In ongoing work, we are continuing to investigate the benefits of interferenceaware routing under a wider range of scenarios.

\subsection{Convergence of Upper and Lower Bounds}

In most of the previous results in this section, the upper and the lower bounds converged, assuring us of the optimality of the solution. When they did not converge, e.g., Figure 5, we were able to assure ourselves of optimality of the lower bound by manual verification. In general, however, the bounds may not converge, as there is no guarantee that even after adding all the clique constraints the upper bound will be schedulable. This leads to the question: how do we decide when to stop looking for even tighter bounds? Given that the conflict graph may

\begin{tabular}{|r|r|r|r|}
\hline Grid Size & Lower Bound & Upper Bound & Time \\
\hline $3 \times 3$ & 0.25 & 0.25 & 2 \\
\hline $5 \times 5$ & 0.5 & 0.5 & 2 \\
\hline $7 \times 7$ & 0.495 & 0.5 & 25 \\
\hline $9 \times 9$ & 0.474 & 0.5 & 35 \\
\hline $11 \times 11$ & 0.479 & 0.5 & 40 \\
\hline
\end{tabular}

Table 5: Lower and upper bounds after 150,000 units of effort. Time in minutes.

\begin{tabular}{|r|r|r|r|}
\hline Effort & Lower Bound & Upper Bound & Time \\
\hline 10000 & 0.443 & 0.5 & 2 \\
\hline 50000 & 0.48 & 0.5 & 5 \\
\hline 100000 & 0.49 & 0.5 & 13 \\
\hline 150000 & 0.495 & 0.5 & 25 \\
\hline 200000 & 0.5 & 0.5 & 41 \\
\hline
\end{tabular}

Table 6: Lower and upper bounds after varying effort for a $7 \times 7$ grid. Time in minutes.

have an arbitrarily complex structure, we cannot wait until the upper and lower bounds are within a small percentage of each other since this may never happen. Even after all the cliques are found, the upper bound may still be well above the optimal feasible solution. Thus, there is no easy way to decide when to stop the calculations. The data we present next does indicate, however, that convergence is quite good in many scenarios.

\subsection{Computational Costs}

In Section 4.1, we mentioned that the effort metric provides only a rough indication of the computational costs of finding the bounds. We now provide more data in this regard. Note that much of the data provided is for the MATLAB [21] solver to which we had ready access; as noted below, the CPLEX [6] solver reduced the computation time by a factor of 7 , albeit on a somewhat faster CPU. Unfortunately, we only had limited access to the CPLEX resource and were able to use it for only a few of our experiments. So it is important to note that there is the potential for significant improvements over the computational costs (for MATLAB) reported here.

In Table 5, we consider the relationship between the size of the network and the amount of time required to compute upper and lower bounds. The table shows the bounds computed after 150,000 units of efforts for several grid sizes, and the time required to compute them. In 


\begin{tabular}{|r|r|r|r|}
\hline Flows & Lower Bound & Upper Bound & Time \\
\hline 2 & 0.578 & 0.583 & 34 \\
\hline 3 & 0.707 & 0.75 & 31 \\
\hline 4 & 0.758 & 0.833 & 29 \\
\hline 5 & 0.799 & 0.875 & 31 \\
\hline 6 & 0.849 & 0.925 & 34 \\
\hline 7 & 0.861 & 1.00 & 36 \\
\hline
\end{tabular}

Table 7: 7x7 grid, multiple flows, 150,000 units of effort. Time in minutes.

\begin{tabular}{|r|r|r|r|}
\hline Flows & Lower Bound & Upper Bound & Time \\
\hline 6 & 0.849 & 0.925 & 5 \\
\hline 7 & 0.861 & 1.00 & 5 \\
\hline
\end{tabular}

Table 8: 7x7 grid, multiple flows, 150,000 units of effort, with CPLEX. Time in minutes.

each case, there is a single flow in the network, with its source and destination nodes at diagonally opposite corners of the grid. The rest of the parameters are similar to those used to plot Figure 5. Note that the upper and lower bounds are not equal in all cases (but they are all close), which indicates that we might not have found the optimal solution in all cases. The computations were done using MATLAB 6.1 [21], on a machine with $1.7 \mathrm{Ghz}$ Pentium processor, and 1.7GB of RAM.

In Table 6, we consider the relationship between the amount of effort, and the closeness of upper and lower bounds, as well as the time required to compute those bounds. The results are based on the $7 \times 7$ grid, with rest of the parameters similar to those used for Table 5. As we discussed in Section 4.1, with more effort, we are likely to add more variables as well as more restrictive constraints in the linear program. So the bounds become tighter.

In Table 7, we consider the relationship between the number of flows in the network, and the amount of time required to compute bounds for a given amount of effort. The results are based on a $7 \times 7$ grid, with multiple flows. For each flow, the source is in the bottom row of the grid, and it communicates with a destination located in the same column, but in the top row. All other parameters are the same as Table 5.

The software used to solve the linear program is also a significant factor in the amount of time required to find the optimal solution. In Table 8, we show the amount of time taken by CPLEX [6] to solve the $7 \times 7$ grid case, with 6 and 7 flows on a 2.7GHz Pentium machine, with 3.7GB of RAM. While we can not compare these entries directly with the corresponding entries in Table 8 , as the machines used to run MATLAB and CPLEX are different, the speedup is still quite significant: a reduction by a factor of 7, from 34-36 minutes down to $5 \mathrm{~min}$ utes. Moreover, MATLAB cannot solve the Mixed Integer Programs resulting from the formulation of singlepath routing. We could only solve these using CPLEX. Unfortunately, we only had limited access to the CPLEX software, so we are unable to report the full set of numbers for CPLEX.

Since these numbers are based on a single run, and are based only on grid graphs, which have a regular connectivity pattern, we cannot draw general conclusions from them. However, some trends are useful to note. We observe that for grid networks, the amount of time required to solve the problem increases with the number of nodes. We also see that for a given effort level, the time required to compute the bounds does not depend significantly on the number of flows in the network. However, the difference between the upper and lower bounds for a given amount of effort tends to increase with increase in the number of flows.

In case of irregular graphs, such as the neighborhood graph shown in Figure 7, we have observed that the amount of time required to solve depends significantly on connectivity and interference patterns.

Finally, we note that we have not included any results involving physical model of communication in this section. We have also not included results that demonstrate the use of links of different capacities. While we have solved such networks (physical models of interference, links of different capacities etc.), we could not do a detailed study due to resource constraints. Therefore, we have chosen to focus on the protocol model of interference in this section.

\section{Dealing with Node Mobility}

So far in this paper, we have assumed that all the nodes in the wireless network are static. we now discuss some possible ways to incorporate node mobility in our model. One way is to repeat the whole process all over: reconstruct connectivity graph and conflict graph based on the new topology, and solve the resulted LP problem. A more efficient way is to take advantage of the efforts 
spent for the old topology, and incrementally compute new bounds. We propose the following incremental approach for the protocol model.

When a node moves from one place to another, it results in adding and/or removing links in a connectivity graph. For ease of discussion, suppose a link $A B$ is removed, and a link $A C$ is added as a result of node $A$ 's movement. The following discussions can be easily generalized to the case when more than one link is added or removed.

The above change in the connectivity graph leads to the following changes in the conflict graph: (i) adding link $A C$ in the connectivity graph results in adding its corresponding vertex to the original conflict graph; in addition, we need to identify the links that conflict with the new link $A C$, and add edges to the original conflict graph to reflect these conflicts; and (ii) removing link $A B$ in the connectivity graph results in deleting its corresponding vertex and all its incident edges from the original conflict graph.

Based on the above changes in the conflict graph, we can incrementally update the independent set constraints to derive a new lower bound as follows. To account for the addition of link $A C$ in connectivity graph, we add independent set constraints that contain $A C$ to the original LP. To account for the deletion of link $A B$, we remove independent set constraints containing $A B$ from the original LP. If the topology change does not cause changes to the objective function (i.e., links between sources and their neighbors remain the same or links between sinks and their neighbors remain the same), then LP solvers, such as lp_solve_inc [16], can take advantage of incremental changes in the linear constraints, and more efficiently derive solution to the new LP than solving it starting from scratch.

Similarly, to derive a new upper bound, we incrementally update the clique constraints as follows. To account for the addition of link $A C$, we add clique constraints involving $A C$ to the original LP; to account for the deletion of link $\mathrm{AB}$, we remove clique constraints involving $A B$ from the original LP. As before, as long as the topology changes do not affect the objective function, LP solvers can more efficiently derive a solution to the new LP based on the incremental changes in the linear constraints.

Incrementally computing the lower and upper bounds is hard under the physical model, because in extreme a node's movement can affect noise level experienced by all nodes, thereby having a global impact on the conflict graph. We plan to further investigate this problem as part of our future work.

\section{Discussion of Limitations}

Our results have demonstrated the flexibility of our model and methodology for computing throughput bounds. However, our work does have some limitations, as we discuss below.

Time-varying channels pose a problem for our model. Time-varying channel characteristics could result either from the interference caused by other nodes or from physical effects, e.g. mobility-induced fading. Our model does account for fluctuations in the noise level at a node due to the interfering transmissions of other nodes. However, it does not accommodate fluctuations caused by phenomena such as fading. As with mobility, it may be feasible to recompute from scratch if the fluctuations happen slowly.

The computational cost numbers presented in Section 4.5 suggest that our methodology is feasible for modest sized networks of the order of a few hundred nodes, which may be typical of a neighborhood wireless network. However, the methodology in its current form is likely to be too expensive for large-scale networks containing thousands or millions of nodes, e.g. sensor networks. Since energy consumption rather than throughput is often the metric of interest in such large-scale networks, this limitation may be moot.

\section{Conclusion and future work}

In this paper we have presented a model and methodology for computing bounds on the optimal throughput that can be supported by a multi-hop wireless network. A key distinction compared to previous work is that we work with any given wireless network configuration and workload specified as inputs. No assumptions are made on the homogeneity of nodes with regard to radio range or other characteristics, or regularity in communication pattern. We use a conflict graph to model wireless interference under various conditions (multiple radios, multiple channels, etc.). We view the generality of our methodology and the conflict graph framework as a key contribution of our work.

Although the bounds that we compute on the optimal throughput assume the ability to finely control and care- 
fully schedule packet transmissions, the optimal routes yielded by our analysis often outperform shortest path routes even under "real-world" conditions such as uncoordinated scheduling and MAC contention. In ns-2 simulations, we have observed a throughput improvement of over a factor of 2 in some cases. The reason for this significant improvement is that the optimal routes often tend to be less interference-prone than the default shortest path routes.

We have also considered the impact of new nodes on the per-node throughput in multi-hop wireless networks. Contrary to previous results, we have found that the addition of new nodes can be beneficial for all nodes, under the (realistic) assumption that each node is active for only a small fraction of the time. The richer connectivity enabled by new nodes presents increased opportunities for routing around interference "hotspots" in the network. This more than offsets the increase in traffic load caused by the new nodes.

In ongoing work, we are continuing to investigate the benefits of interference-aware routing under a wide range of scenarios. Our next step after that would be to design a practical interference-aware routing protocol, which addresses interesting challenges such as constructing the conflict graph and computing optimal routes in a distributed manner.

\section{Acknowledgments}

We thank Jayavel Shanmugasundaram and László Lovász for helpful discussions. We also thank Jon Howell for his helpful comments on earlier drafts of this paper.

\section{References}

[1] N. Abramson. THE ALOHA SYSTEM - Another Alternative for Computer Communications". Proc. AFIPS Fall Joint Computer Conference, pages 281-285, 1970.

[2] Bay area wireless users group. http://www.bawug.org/.

[3] M. Berkelaar. lp_solve: linear programming code. ftp://ftp.ics.ele.tue.nl/pub/lp_solve/.
[4] V. ChavtaL. Linear Programming. W. H. Freeman and Compnay, 1983.

[5] D. S. J. D. Couto, D. Aguayo, B. A. Chambers, and R. Morris. Performance of multihop wireless networks: Shortest path is not enough. In 1st Workshop on Hot Topics in Networks, Oct. 2002.

[6] Ilog cplex suite, 2003. http://www.ilog.com/products/cplex/.

[7] J. Edmonds. Maximum matching and a polyhedron with 0,1-vertices. Journal of Research of the National Bureau of Standards, B69:125-130, 1965.

[8] D. Estrin, R. Govindan, J. Heidemann, and S. Kumar. Next century challenges: Scalable coordination in sensor networks. In ACM MOBICOM, Aug. 1999.

[9] M. R. Garey and D. S. Johnson. Computers and Intracctability: A guide to the theory of NP completness. W. H. Freeman and Company, 1979.

[10] M. Gastpar and M. Vetterli. On the capacity of wireless networks: the relay case. In IEEE INFOCOM, Jun. 2002.

[11] M. Grossglauser and D. Tse. Mobility increases the capacity of ad-hoc wireless networks. In IEEE INFOCOM, Apr. 2001.

[12] M. Grotschel, L. Lovasz, and A. Schrijver. The ellipsoid method and its consequences in combinatorial optimization. Combinatorica, 1:169-197, 1981.

[13] M. Grotschel, L. Lovasz, and A. Schrijver. Geometric Methods in Combinatorial Optimization. Academic Press, 1984.

[14] P. Gupta and P. R. Kumar. The capacity of wireless networks. IEEE Transactions on Information Theory, 46(2), Mar. 2000.

[15] P. Gupta and P. R. Kumar. The capacity of wireless networks. IEEE Transactions on Information Theory, 46(2):388-404, Mar. 2000.

[16] R. Haemmerlé. Lp solve - incremental version. http://contraintes.inria.fr/haemmerl/lp_solve_inc/.

[17] D. B. Johnson and D. A. Maltz. Dynamic source routing in ad-hoc wireless networks. In T. Imielinski and H. Korth, editors, Mobile Computing. Kluwer Academic Publishers, 1996

[18] L. G. Khachiyan. A polynomial algorithm in linear programming. Soviet Mathematics Doklady, 20:191-194, 1979.

[19] M. Kodialam and T. Nandagopal. Charaterizing achievable rates in multi-hop wireless newtorks: The joint routing and scheduling problem. In ACM MOBICOM, Sep. 2003. 
[20] J. Li, C. Blake, D. S. J. D. Couto, H. I. Lee, and R. Morris. Capacity of ad hoc wireless networks. In ACM MOBICOM, Jul. 2001.

[21] Matlab version 6.1. http://www.matlab.com/.

[22] M.Chudnovsky, N. Robertson, P.D.Seymour, and R.Thomas. The Strong Perfect Graph Theorem. Submitted for publication., February 2003.

[23] T. Nandagopal, T. Kim, X. Gao, and V. Bharghavan. Achieving mac layer fairness in wireless packet networks. In ACM MOBICOM, Aug. 2000.

[24] Ns-2 (network simulator), $1995 . \quad$ http://wwwmash.cs.berkeley.edu/ns/.

[25] V. D. Park and M. S. Corson. A highly adaptive distributed routing algorithm for mobile wireless networks. In Proc. of IEEE INFOCOM'97, Apr. 1997.

[26] C. E. Perkins and P. Bhagwat. Highly dynamic destination-sequenced distance vector routing ( $\mathrm{dsdv}$ ) for mobile computeres. In Proc. of ACM SIGCOMM'94, Sep. 1994.

[27] C. E. Perkins and E. M. Royer. Ad-hoc on-demand distance vector routing. In Proc. of IEEE WMCSA'99, Feb. 1999.

[28] Seattle wireless. http://www.seattlewireless.net/.

[29] X. Yang and N. H. Vaidya. Priority scheduling in wireless ad hoc networks. In ACM MobiHoc, June 2002.

\section{A Proof of Theorem 1}

Suppose we are given a graph $G$ and we want to compute the cardinality of its maximum independent set. We now construct a wireless network such that the optimal throughput it can support under the protocol interference model is the same as the cardinality of the maximum independent set of $G$. Create two wireless nodes, a source $s$ and a receiver $r$. For every vertex in $G$ add a wireless link of unit capacity between $s$ and $r$. For every edge between two nodes in $G$, assume a conflict between the corresponding wireless links in the network. (Such a network may arise, for instance, if nodes $s$ and $r$ are each equipped with multiple radios set either to the same (i.e., interfering) channel or to separate (i.e., non-interfering) channels. It is not hard to see that the optimal throughput is achieved if and only if a maximum independent set in $G$ is scheduled. Thus finding the optimal throughput of the wireless network is equivalent to finding the cardinality of the maximum independent set of graph $G$, which is known to be a hard problem.

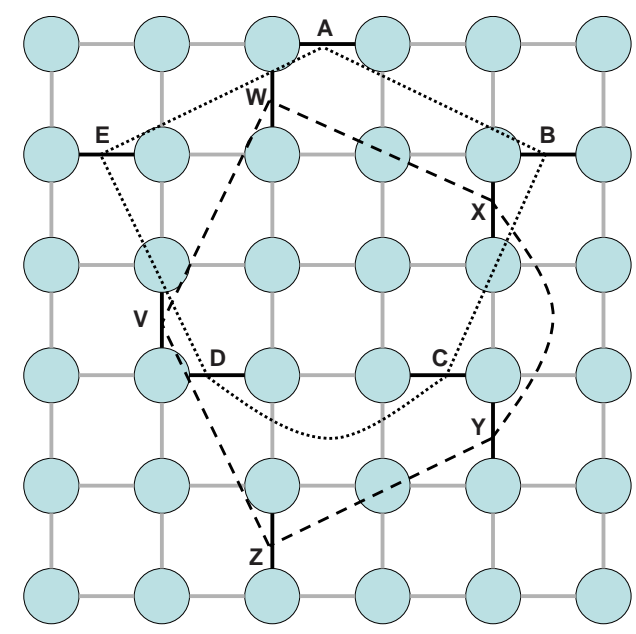

Figure 11: A 6x6 grid connectivity graph. ABCDE and VWXYZ are examples of odd holes in the corresponding conflict graph, assuming an 802.11-style MAC, communication range equal to the lateral spacing between neighbors, and interference range equal to twice the communication range. These odd holes also happen to be odd anti-holes.

The above proof may come across as contrived since the wireless network we constructed is unlikely to arise in practice. This raises an interesting question of whether realistic wireless networks could give rise to complex conflict graphs? Our answer is both yes and no. Our answer is "yes" because the maximum independent set problem is hard due to the existence of odd holes and odd anti-holes in the given graph ${ }^{2}$. As shown in Figure 11, very realistic and simple grid graphs could have conflict graphs with many odd holes and odd anti-holes. On the other hand, our answer is "no" because realistic conflict graphs may have some special property or structure that could make the problem of finding the maximum independent set easy. We have been unable to identify any such property, but our failure does not mean that no such property exists (though the complex conflict graphs arising from the simple grid graphs, as in Figure 11, diminish our optimism). In view of this, we believe that the heuristic approach presented in Section 3 is reasonable. In the following subsection, we discuss certain special cases in which optimal solution may be found in polynomial time.

\footnotetext{
${ }^{2}$ If a graph does not have any odd holes or anti-holes then the graph is termed perfect [22], and for perfect graphs there are polynomial time algorithms to solve the maximum independent set problem [12].
} 


\section{B Polynomial Time Algorithm in Spe- cial Cases}

Even in special cases where polynomial time algorithms may exist, they may be too expensive to be of practical interest. One such special case arises in the context of grid graphs when the conflict radius is zero. By zero conflict radius we mean that two links conflict if and only if they share an endpoint. In this simple and somewhat unrealistic setting, the conflict graph is nothing but the line graph of the underlying grid network. The line graph, $L(G)$, of a graph, $G$, is a graph on the edges of $G$, i.e., the vertices of $L(G)$ correspond to the edges of $G$. There is an edge between two vertices of $L(G)$ if the corresponding edges in $G$ have a vertex in common.

Note that we have assume that our network in this case is a grid. A grid is a bipartite graph, and bipartite graphs are perfect. The line graph of a perfect graph is perfect too. Hence the conflict graph of a grid graph with a zero conflict radius is a perfect graph. A perfect graph has the property that its set of clique constraints define its independent set polytope. So if we write a linear program with all the clique constraints together with the flow constraints then we can find the optimal network throughput. The problem, however, is that the number of cliques could still be exponentially many. (Although this does not happen with grid graphs, it could very well happen with other perfect graphs.) A solution is to use the ellipsoid algorithm [18] to optimize linear functions over a polytope. This algorithm does not require all the constraints in an explicit form to optimize a linear function over a polytope, hence we do not have to enumerate the exponentially many clique constraints. The ellipsoid algorithm only needs a subroutine that given a potential solution indicates whether the constraints are satisfied or not, and if not identifies at least one constraint which is not satisfied. Such a subroutine is called separation oracle. The separation oracle for our problem would be one that finds a violated clique constraint given a usage vector. This can be accomplished using the Grotschel semidefinite programming algorithm for finding the heaviest clique [13]. However, both the ellipsoid algorithm and the semidefinite algorithm have a running time of $O\left(n^{3}\right)$, so in combination their running time is $O\left(n^{6}\right)$. Thus this polynomial time algorithm is not very practical.

As discussed in Section 2, Kodialam and
Nandagopal [19] present an approximation algorithm for a similar case. They also assume zero conflict radius but the underlying conflict graph can be arbitrary graphs instead of a grid graph. We note that our algorithm still finds the optimal solution for the problem within polynomial time. Since the conflict radius is zero, conflict graph is just a line graph of the connectivity graph. Independent set polytope of the conflict graph is just the matching polytope of the connectivity graph. Edmonds [7] gave a linear program describing the matching polytope of an arbitrary graph. Hence, for this problem, we can describe the independent set polytope by a linear program. This implies that our algorithm can compute the optimal solution for the case when the conflict radius is zero.

\section{Theoretical limits on the upper- bound}

One of the questions, which our upper-bounding heuristic raises, is how good is the upperbound if we include all the clique constraints, which can itself take exponential computation time. We have given examples (odd holes) earlier which show that unlike the lowerbound, the upperbound may not always be tight. The next question is, is the upperbound even likely to be within any constant factor of the optimal. Unfortunately, the answer can be no. Consider the two nodes example discussed in Appendix A. Let the conflict graph be $G$. We have already shown that the maximum throughput from $s$ to $r$ is the cardinality of the maximum independent set in $G$. From probabilistic graph theory we know that there are triangle-free graphs on $n$ nodes which have independent size of $O(\sqrt{n})$. Suppose the conflict graph $G$ is one of those graphs. Since $G$ is triangle-free, only clique constraints are edges. So using each wireless link for half the time satisfies all the cliques, the upperbound can't be better that $n / 2$, whereas the optimal is $O(\sqrt{n})$. So the upperbound is not within any constant factor of optimum throughput.

The next question, then, is whether the quality of the upperbound improves when we add all the odd-hole and the odd-anti-hole constraints. We do not explore this question, but do believe that the upperbound will not be within any constant factor even then. The reason for our belief is that the stable set polytope can include complicated structures such as odd wheels, that will need to be 
considered even after all odd-hole and odd anti-hole constraints have been discovered. Instead, we now present a technique that may improve the convergence rate of our algorithm, but does not guarantee tightness.

The technique is a separation oracle that given a conflict graph $G$ and a candidate solution $\lambda$ finds a violated odd hole constraint, if any. Such an oracle could be used to improve the convergence rate of the algorithm presented in Section 3. Note that this separation oracle is applicable to general graphs; for the perfect conflict graph considered in Section B above, there are no odd holes anyway.

Consider an odd hole, $H$, of the given conflict graph $G$. Any vector $\lambda$ inside the independent set polytope of $G$ must satisfy the following: $\sum_{i \in H} \lambda_{i} \leq(|H|-1) / 2$. A violated odd hole is one for which this constraint is not satisfied. Before attempting to find a violated odd hole, we may assume that the given $\lambda$ satisfies all the edge constraints, i.e., $\lambda_{i}+\lambda_{j} \leq 1$ for every edge in $G$, because if it does not then we can include the violated edge constraint to shrink the upperbounding polytope. After making this assumption we define a weight function on the edges. For every edge $i j$ of the graph $G$, we define its weight to be $1-\lambda_{i}-\lambda_{j}$, which is guaranteed to be nonnegative. With this weight function we find the lightest (i.e., least-weight) odd cycle in the graph. The lightest odd cycle can be found using a bipartite graph construct as explained in the next paragraph. Let $C$ be the lightest odd cycle. $\sum_{i j \in C}\left(1-\lambda_{i}-\lambda_{j}\right)<1$ is equivalent to $\sum_{i \in C} \lambda_{i}>\frac{|C|-1}{2}$. So, if the weight of the lightest odd cycle is less than 1 then the cycle is a violated odd hole. If the weight of the lightest odd cycle is 1 or more then there is no violated odd hole.

Now we come to the question of efficiently finding the lightest odd cycle. Let $G$ be the graph in which we need to find the lightest odd cycle. We construct a bipartite graph, $B$, as follows. For every vertex $v$ in $G$ we put two vertices $v_{l}$ and $v_{r}$ in $B$ (the subscripts $l$ and $r$ can conceptually be thought of as representing the left and right "halves" of the the bipartite graph $B$ ). For every edge $u v$ in $G$ we put two edges $u_{l} v_{r}$ and $u_{r} v_{l}$ in $B$. Now an odd cycle in $G$ becomes an odd length path in $B$ e.g., $u v w u$ becomes $u_{l} v_{r} w_{l} u_{r}$. So for every vertex $u$ in $G$ we find the shortest path from $u_{l}$ to $u_{r}$ in $B$. The shortest such path in $B$ yields the lightest odd cycle in $G$. 